\title{
IC 4499 revised: Spectro-photometric evidence of small light-element variations
}

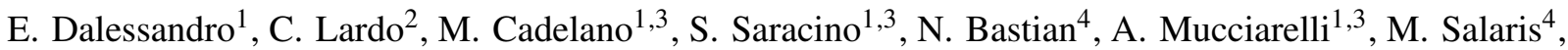 \\ P. Stetson ${ }^{5}$, and E. Pancino 6,7 \\ 1 INAF - Astrophysics and Space Science Observatory Bologna, Via Gobetti 93/3, 40129 Bologna, Italy \\ e-mail: emanuele.dalessandro@inaf.it \\ 2 Laboratoire d'Astrophysique, Ecole Polytechnique Fédérale de Lausanne, Observatoire de Sauverny, 1290 Versoix, Switzerland \\ 3 Dipartimento di Fisica e Astronomia, Via Gobetti 93/2, 40129 Bologna, Italy \\ 4 Astrophysics Research Institute, Liverpool John Moores University, 146 Brownlow Hill, Liverpool L3 5RF, UK \\ 5 Herzberg Astronomy and Astrophysics, National Research Council Canada, 5071 West Saanich Road, \\ Victoria, BC V9E 2E7, Canada \\ ${ }^{6}$ INAF - Osservatorio Astrofisico di Arcetri, Largo Enrico Fermi 5, 50125 Firenze, Italy \\ 7 ASI Science Data Center, Via del Politecnico SNC, 00133 Rome, Italy
}

Received 15 June 2018 / Accepted 16 July 2018

\begin{abstract}
It has been suggested that IC 4499 is one of the very few old globulars to not host multiple populations with light-element variations. To follow-up on this very interesting result, here we have made use of accurate HST photometry and FLAMES at VLT high-resolution spectroscopy to investigate in more detail the stellar population properties of this system. We find that the red giant branch of the cluster is clearly bimodal in near-UV-optical colour-magnitude diagrams, thus suggesting that IC 4499 is actually composed by two sub-populations of stars with different nitrogen abundances. This represents the first detection of multiple populations in IC 4499. Consistently, we also find that one star out of six is Na-rich to some extent, while we do not detect any evidence of intrinsic spread in both $\mathrm{Mg}$ and $\mathrm{O}$.

The number ratio between stars with normal and enriched nitrogen is in good agreement with the number ratio - mass trend observed in Galactic globular clusters. Also, as typically found in other systems, nitrogen rich stars are more centrally concentrated than normal stars, although this result cannot be considered conclusive because of the limited field of view covered by our observations $\left(\sim 1 r_{\mathrm{h}}\right)$. On the contrary, we observe that both the RGB UV colour spread, which is a proxy of $\mathrm{N}$ variations, and Na abundance variations, are significantly smaller than those observed in Milky Way globular clusters with mass and metallicity comparable to IC 4499. The modest $\mathrm{N}$ and $\mathrm{Na}$ spreads observed in this system can be tentatively connected to the fact that IC 4499 likely formed in a disrupted dwarf galaxy orbiting the Milky Way, as previously proposed based on its orbit.
\end{abstract}

Key words. globular clusters: general - globular clusters: individual: IC 4499 - Hertzsprung-Russell and C-M diagrams techniques: photometric - stars: abundances -techniques: spectroscopic

\section{Introduction}

Globular clusters (GCs) exhibit intrinsic star-to-star variations in their light element content. In fact, while some GC stars have the same light-element abundances as the field at the same metallicity (first population or generation-FP), others show enhanced $\mathrm{N}$ and $\mathrm{Na}$ along with depleted $\mathrm{C}$ and $\mathrm{O}$ abundances (second population or generation-SP). The manifestation of such light-element inhomogeneities is referred to as multiple populations (MPs). A number of scenarios have been proposed over the years to explain the formation of MPs (e.g. Decressin et al. 2007; D'Ercole et al. 2008; Bastian et al. 2013; Denissenkov \& Hartwick 2014; D'Antona et al. 2016; Gieles et al. 2018), however, their origin is still strongly debated.

Nearly all massive and old Galactic GCs host MPs (e.g., Piotto et al. 2015; Milone et al. 2017; Bragaglia et al. 2017). In addition, MPs are also found in the Magellanic Clouds stellar clusters (Mucciarelli et al. 2009; Dalessandro et al. 2016), in GCs in dwarf galaxies such as Fornax (Larsen et al. 2012, 2018), in the M31 GC system (Schiavon et al. 2013) and there are strong indications (though indirect) that they are ubiquitous in stellar clusters in massive elliptical galaxies (e.g., Chung et al. 2011).

However, it is currently unclear which GC property drives the onset of MPs in GCs. One property that has a strong effect on both the relative fraction of SP stars as well as the extent of the abundance spreads is the GC present-day mass (e.g., Carretta et al. 2010; Schiavon et al. 2013; Milone et al. 2017). Low mass GCs $\left(<10^{5} M_{\odot}\right)$ have $\sim 50 \%$ fractions of SP stars increasing to $\sim 90 \%$ in the most massive GCs $\left(\sim 10^{6} M_{\odot}\right.$; Milone et al. 2017). By extrapolating this relation to the open cluster mass regime $\left(\sim 10^{3}-10^{4} M_{\odot}\right)$, we would expect to find $1-10 \%$ of SP, but they have not been found in these system.

Cluster age turns out to be another important parameter. Indeed, MPs have been found in intermediate age (2-8 Gyr) clusters in the Magellanic Clouds (Niederhofer et al. 2017; Martocchia et al. 2018). Conversely, massive $\left(\sim 10^{5} M_{\odot}\right)$ clusters younger than $\sim 2 \mathrm{Gyr}$ do not show any inhomogeneity in their light-element content or any photometric signatures that can be indicative of chemical anomalies (e.g. Mucciarelli et al. 2008; Martocchia et al. 2018). 
Only very few exceptions to this general emerging scheme are known in the literature. One of them is the ancient $\left(t_{\mathrm{age}}=12 \pm 0.75\right.$ Gyr; Dotter et al. 2011) GC IC 4499(Walker et al. 2011), other classical cases are Ruprecth 106 (Villanova et al. 2013) and E3 (Monaco et al. 2018). IC 4499 has been reported to not host MPs based on ground based multi-band photometry by Walker et al. (2011). Given the age and mass $\left(M_{\mathrm{V}}=-7.32\right.$; Harris 1996 - edition 2010) of this cluster, the lack of MPs is totally unexpected and extremely interesting. In fact, finding clusters that do not host MPs may lead to new insights into GC formation.

Here we have used Hubble Space Telescope (HST) imaging targeting the cluster central regions and FLAMES at VLT highresolution spectroscopy of six bright cluster members to conclusively establish whether IC 4499 hosts MPs or not. The paper is structured as follows. In Sect. 2 the photometric observational database and analysis are presented. In Sect. 3 we detail on the spectroscopic analysis. In Sect. 4 the results obtained by means of the photometric and spectroscopic data sets are discussed. In Sect. 5 we summarise the most relevant results and draw our conclusions.

\section{Photometric observations and data analysis}

\subsection{Photometric data and reduction}

This work is based on two HST data-sets. One is composed of near-UV and optical images obtained through the Wide Field Camera 3 (WFC3) UVIS channel (GO 14723, PI: Dalessandro). Eight images were acquired with the F275W filter and exposure time $t_{\exp }=1100 \mathrm{~s}$, four F336W images with $t_{\exp }=650 \mathrm{~s}$ and additional four exposures acquired with the $\mathrm{F} 438 \mathrm{~W}$ band and $t_{\exp }=100 \mathrm{~s}$. The other programme consists of five images acquired with the F606W filter (one with $t_{\exp }=60 \mathrm{~s}$ and four with $t_{\exp }=603 \mathrm{~s}$ ) and five F814W images (one with $t_{\exp }=65 \mathrm{~s}$ and four with $t_{\exp }=636 \mathrm{~s}$ ) obtained with the Wide Field Camera of the Advanced Camera for Surveys (GO: 11586, PI: Dotter). In both data-sets, an appropriate dither pattern of a few arcseconds has been adopted for each pointing in order to fill the inter-chip gaps and avoid spurious effects due to bad pixels. The field of view (FOV) covered by the two datasets is shown in Fig. 1.

The photometric analysis has been initially performed independently for each data-set, camera and chip by using DAOPHOT IV (Stetson 1987). Tens of bright and isolated stars have been selected in each frame to model the point spread function (PSF), which has been eventually applied to all sources detected in each image above $5 \sigma$, where $\sigma$ is the standard deviation of the background counts. We then created a master catalogue composed of stars detected in at least half of the images for each available filter and two bands. At the corresponding positions of stars in this final master-list, a fit was forced with DAOPHOT/ALLFRAME (Stetson 1994) in each frame of the two data-sets. For each star thus recovered, multiple magnitude estimates obtained in each chip were homogenised by using DAOMATCH and DAOMASTER, and their weighted mean and standard deviation were finally adopted as star magnitude and photometric error.

Instrumental magnitudes of both the WFC3 and ACS catalogues were reported to the VEGAMAG photometric system. For the case of the WFC3, we used the equations and zero-points reported in the HST web page, while for the ACS we used the stars in common with the catalogue used by Dotter et al. (2011) and publicly available in the ACS Survey of Galactic Globular Clusters page ${ }^{1}$. The resulting $\left(m_{\mathrm{F} 275 \mathrm{~W}}, m_{\mathrm{F} 275 \mathrm{~W}}-m_{\mathrm{F} 438 \mathrm{~W}}\right)$ colour

\footnotetext{
1 http://www.astro.ufl .edu/ ata/public_hstgc/
} databases.html

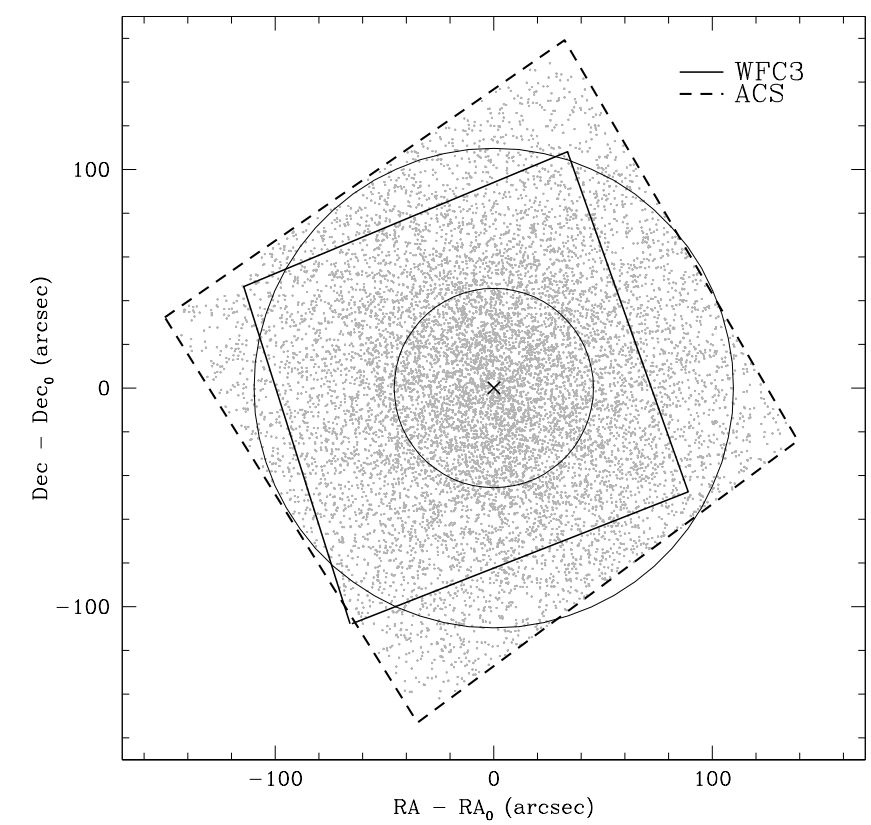

Fig. 1. Map of the HST/WFC3 and HST/ACS images FOVs. The two black circles represent the cluster core and half mass radii from Harris (1996).

magnitude diagram (CMD) is shown in Fig. 2. Instrumental coordinates were reported to the absolute coordinate system $(\alpha, \delta)$ by using the stars in common with the Dotter et al. (2011) catalogue as secondary astrometric standards and by means of the software CataXcorr ${ }^{2}$.

\subsection{Relative proper motion}

By taking advantage of the large temporal baseline existing between the WFC3 and ACS data-sets $(\Delta T=6.915 \mathrm{yr})$, we performed a relative proper motion (PM) analysis to clean the cluster CMD from field interlopers. We note that IC 4499 is at heliocentric distance $d=18.8 \mathrm{Kpc}$ thus the PM information coming from the Gaia Data Release 2 are available only for the brighter portion of the red giant branch and suffer from significant uncertainties.

For the proper motion analysis we adopted the approach described in Dalessandro et al. (2013; see also Massari et al. 2013; Bellini et al. 2014; Cadelano et al. 2017). The procedure consists in measuring the instrumental position displacements of the stars detected in both epochs, once a common distortion-free reference frame is defined. The reference frame adopted in this analysis is the geometric distortion corrected ACS catalogue (see Sect. 2.1).

For each data-set we derived mean instrumental positions $(x, y)$ as the average of the positions of stars detected in half of the entire number of images (see Sect. 2). In the WFC3 case $(x, y)$ have been corrected for geometric distortions by applying the equations published in Bellini \& Bedin (2009) for the $F 275 \mathrm{~W}$ UVIS filter. For the ACS catalogue we adopted the ACS/WFC Distortion Correction Tables (IDCTAB) provided on the dedicated page of the Space Telescope Science Institute.

2 We used CataXcorr, a code which is specifically developed to perform accurate astrometric solutions. It has been developed by P. Montegriffo at INAF - Osservatorio Astronomico di Bologna. This package is available at http://davide2.bo.astro.it/ paolo/ Main/CataPack.html, and has been successfully used in a large number of papers by our group in the past ten years. 


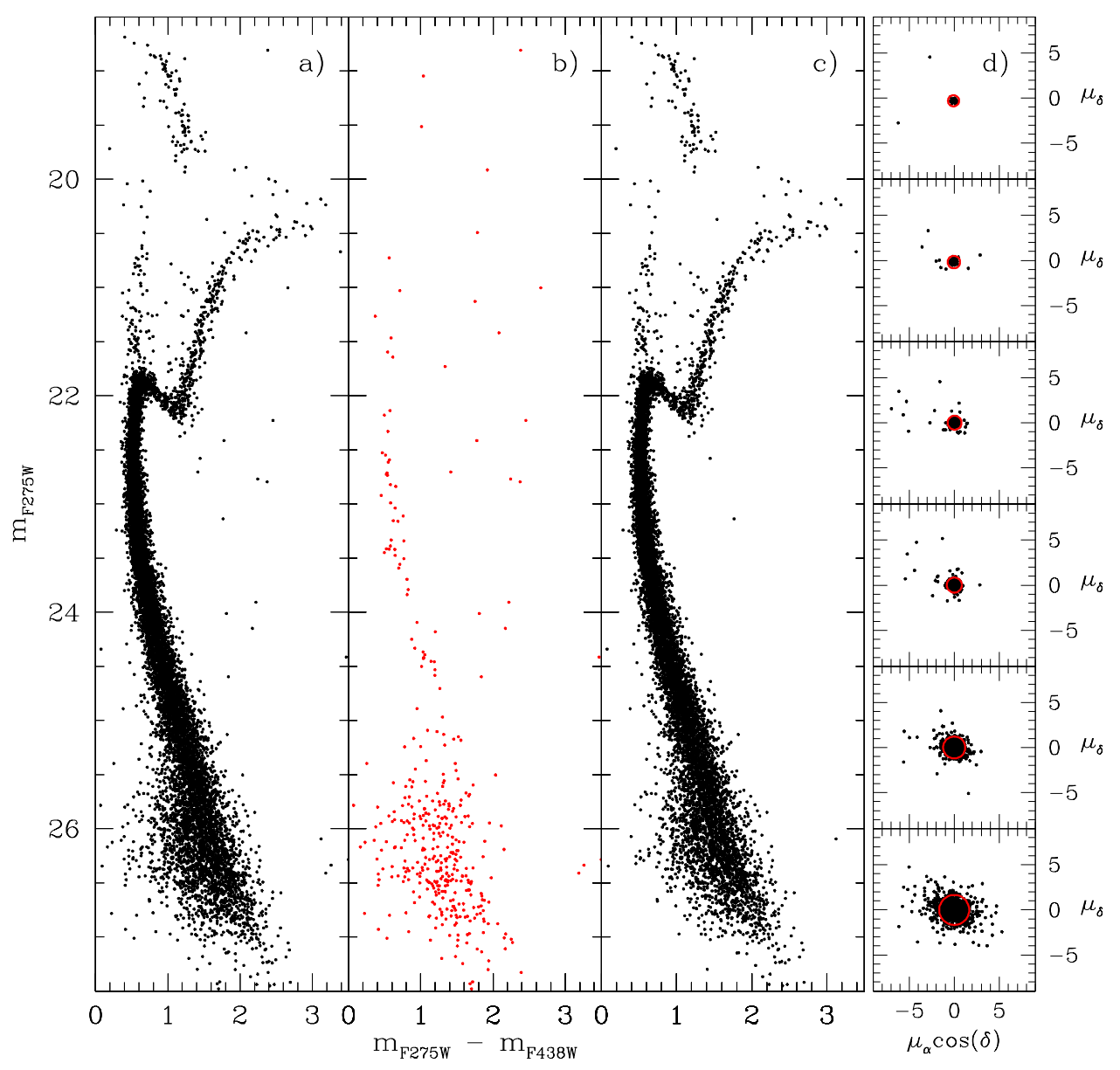

Fig. 2. Panel a: $\left(m_{\mathrm{F} 275 \mathrm{~W}}, m_{\mathrm{F} 275 \mathrm{~W}}-m_{\mathrm{F} 438 \mathrm{~W}}\right)$ $\mathrm{CMD}$ of all the stars in common between the ACS and WFC3 catalogues; panel $b$ : CMD of all the likely contaminating objects as selected from the VPDs; panel c: relative PM-cleaned CMD obtained by using only likely cluster members; panels $d$ : VPDs at different magnitude levels. The solid red circle represents the fiducial region used to select likely cluster members.
We then estimated accurate transformations between the WFC 3 catalogue and the reference frame. To this aim we selected a sample of $\sim 2000$ stars having magnitude $18.0 \leq m_{\mathrm{F} 814 \mathrm{~W}} \leq 20.5$ (corresponding to magnitudes $19.5 \leq m_{\mathrm{F} 336 \mathrm{~W}} \leq 22.0$ ), which are likely cluster members on the basis of their positions in the CMD (stars distributed along the lower RGB, the sub-giant branch and the upper main sequence). We then applied a six-parameter linear transformation between the two epochs, treating each chip of the WFC3 separately, in order to maximise the accuracy.

The derived transformations, have been then applied to all the stars in common between the two catalogues. The relative $\mathrm{PM}$ is finally determined by measuring the difference of the mean $(x, y)$ positions of each star in the two epochs, divided by their temporal baseline. Such displacements are in units of pixels $\mathrm{yr}^{-1}$. We finally converted the PMs into absolute units (mas $\mathrm{yr}^{-1}$ ) by multiplying the measured displacements by the pixel scale of the master frame $\left(0.05^{\prime \prime}\right.$ pixel $\left.^{-1}\right)$.

Results of the PM analysis are shown in Fig. 2. In panel a, the $\left(m_{\mathrm{F} 275 \mathrm{~W}}, m_{\mathrm{F} 275 \mathrm{~W}}-m_{\mathrm{F} 438 \mathrm{~W}}\right) \mathrm{CMD}$ of the stars in common between ACS and WFC3 is shown and the relative PM measurements are shown as vector point diagrams (VPD) in panel d for 6 different bins of $1.5 \mathrm{mag}$ each. A $\sigma$-clipping procedure was applied to separate field stars from cluster members in each VPD. Stars located within the red circles (likely cluster members) are plotted as black points in panel c of Fig. 2, while those located outside the circles (likely field interlopers) are shown as red points in the panel b.

\subsection{Differential reddening}

IC 4499 has a moderately large colour excess $E(B-V) \approx 0.23$ (Harris 1996), hence for the goal of this work it is important

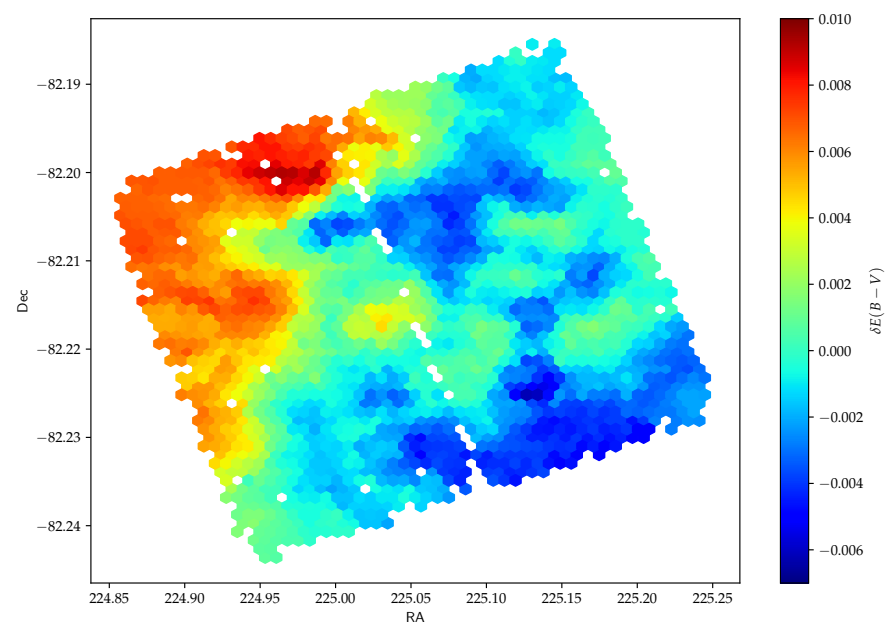

Fig. 3. Differential reddening map of IC 4499 in the ACS+WFC3 FOV obtained as described in Sect. 2.3.

to quantify and eventually correct for any reddening variations within the HST FOV. Differential reddening was estimated by using likely member stars (as constrained from relative PMs analysis described in Sect. 2.2) selected in the magnitude range $18.5 \leq m_{\mathrm{F} 606 \mathrm{~W}} \leq 21.0$. For these stars a mean ridge-line (MRL) in the $\left(m_{\mathrm{F} 606 \mathrm{~W}}, m_{\mathrm{F} 606 \mathrm{~W}}-m_{\mathrm{F} 814 \mathrm{~W}}\right) \mathrm{CMD}$ (Fig. 3) was defined. We then computed the geometric distance $(\Delta X)$ to the MRL for each star within $3 \sigma$ from it, where $\sigma$ is the colour spread around the MRL. By definition the MRL has $\Delta X=0$ and all stars are distributed around this value. Then for each star in the catalogue, differential reddening is estimated by computing the mean of the 


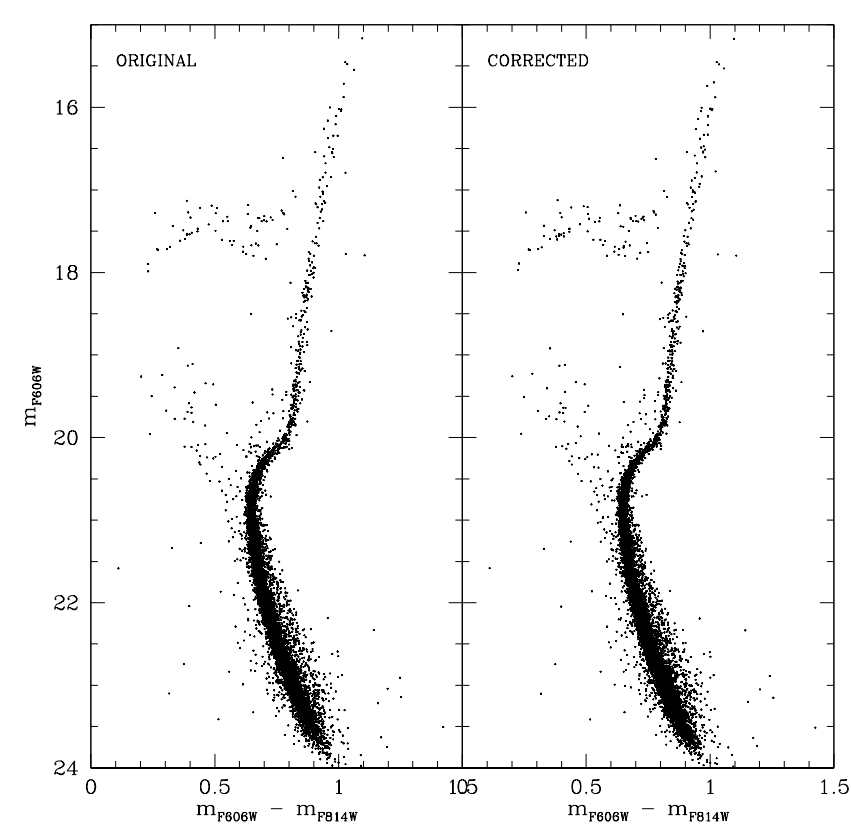

Fig. 4. PM-cleaned $\left(m_{\mathrm{F} 606 \mathrm{~W}}, m_{\mathrm{F} 606 \mathrm{~W}}-m_{\mathrm{F} 814 \mathrm{~W}}\right) \mathrm{CMDs}$ of IC4499 before (left panel) and after (right panel) differential reddening correction.

$\Delta X$ values of the 20 nearest (in space) selected stars. We have verified that in this specific case this number of neighbour stars is the best compromise between good statistics and spatial resolution. Results do not change significantly if a slightly different number of neighbour stars (from ten to 30) is adopted. Finally, $\Delta X$ values are transformed into differential reddening $\delta E(B-V)$ by using the following equation:

$$
\delta E(B-V)=\frac{\Delta X}{\sqrt{2 R_{\mathrm{F} 606 \mathrm{~W}}^{2}+R_{\mathrm{F} 814 \mathrm{~W}}^{2}-2 R_{\mathrm{F} 606 \mathrm{~W}} R_{\mathrm{F} 814 \mathrm{~W}}}}
$$

where $R_{\mathrm{F} 606 \mathrm{~W}}$ and $R_{\mathrm{F} 814 \mathrm{~W}}$ are the exctintion coefficients in the two bands respectively. We adopted $R_{\mathrm{F} 606 \mathrm{~W}}=2.874$ and $R_{\mathrm{F} 814 \mathrm{~W}}=1.884$ as given by Casagrande \& VandenBerg (2014). The resulting reddening distribution within the WFC3ACS FOV is shown in Fig. 3. A maximum $\delta E(B-$ $V) \sim 0.015 \mathrm{mag}$ is found within the HST FOV. Figure 4 shows the $\left(m_{\mathrm{F} 606 \mathrm{~W}}, m_{\mathrm{F} 606 \mathrm{~W}}-m_{\mathrm{F} 814 \mathrm{~W}}\right) \mathrm{CMD}$ of IC 4499 before (left panel) and after the differential reddening corrections have been applied (right panel). In the following analysis we will use PM cleaned and differential reddening corrected CMDs.

\section{Spectroscopic observations and data analysis}

\subsection{Observations and data reduction}

We used FLAMES at VLT observations in the combined MEDUSA+UVES mode (097.D-0111(A) - PI: Bastian), which allows the simultaneous allocation of 8 UVES high-resolution and 132 MEDUSA mid-resolution fibers. Only UVES spectra will be used in this work, while the MEDUSA ones will be presented in a forthcoming paper (Lardo et al., in prep.) devoted to study the metallicity and $\alpha$-element abundance distributions of the IC 4499 stars. UVES spectra were obtained through the 580 Red Arm spectral configuration, providing a spectral resolution of $R \sim 47000[\lambda / \Delta \lambda]$ in the wavelength range between 4800 $6800 \AA$. A total of three exposures of $50 \mathrm{~min}$ each for the same targets configuration has been secured in Service Mode during
July 2016. Targets have been selected along the brightest portion of the RGB ( $V \leq 16 \mathrm{mag}$ ) from the UBVI photometric catalogue by Stetson (1994). Two out of the eight available UVES fibers have been used to sample the sky background and perform a robust sky subtraction for each individual exposure. The basic parameters of the six targets are listed in Table 1, along with other useful information. Data reduction was performed by following the standard recipes provided by the latest version of the ESO UVES pipeline. UVES data were bias subtracted, flat fielded, wavelength calibrated, and extracted using the standard UVES/esorex routines. Spectra were corrected for telluric absorption using a synthetic spectrum as a template. The typical signal-to-noise ratio (S/N) per pixel of spectra is $\sim 20$ at $\sim 5300 \AA$ and $\sim 35$ at $\sim 6300 \AA$.

\subsection{Radial velocity and equivalent width measurements}

Radial velocities and equivalent widths (EWs) were obtained with DAOSPEC (Stetson \& Pancino 2008), run through the interface $4 \mathrm{DAO}^{3}$ (Mucciarelli 2013). Atomic lines used in the analysis were selected from the Vienna Atomic Database (VALD, Kupka et al. 1999).

DAOSPEC (Stetson \& Pancino 2008) automatically performs the line profile fitting adopting a saturated Gaussian function. The FWHM for each spectrum has been estimated iteratively, using the wrapper 4DAO, which automatically configures many of DAOSPEC parameters and provides graphical tools to explore the results. Firstly, we performed an analysis using as input value the nominal FWHM of the grating, leaving DAOSPEC free to re-adjust the FWHM in order to minimise the median value of the residuals distribution. The new value of the FWHM is used by $4 \mathrm{DAO}$ as input value for a new run, until a convergence of 0.3 pixel is reached.

The two UVES arms were analysed separately and the agreement between the two velocity estimates is good $\left(\left\langle v_{\mathrm{r}}^{\text {Low }}-v_{\mathrm{r}}^{\mathrm{Up}}\right\rangle=0.4, \sigma=0.4 \mathrm{~km} \mathrm{~s}^{-1}\right)$. Measurements were based on $\simeq 350$ absorption lines of different elements. The resulting velocities and errors are listed in Table 1 . We measured an average $v_{\mathrm{r}}=37.0 \pm 0.4 \mathrm{~km} \mathrm{~s}^{-1}\left(\sigma=1.1 \mathrm{~km} \mathrm{~s}^{-1}\right)$, that agrees with the value $\left(v_{\mathrm{r}}=31.5 \pm 0.4 \mathrm{~km} \mathrm{~s}^{-1}\right)$ measured by Hankey \& Cole (2011) from their lower resolution spectra $(R \sim 10000)$. All six stars result to be member based on their radial velocities.

\subsection{Abundance analysis}

Chemical abundances for iron and light-elements $(\mathrm{O}, \mathrm{Na}$, and $\mathrm{Mg}$ ) were derived using the GALA (Mucciarelli et al. 2013) package and the classical EW method for abundance analysis. Model atmospheres were calculated with the ATLAS9 code assuming local thermodynamic equilibrium (LTE) and one-dimensional, plane-parallel geometry; starting from $\alpha$-enhanced grid of models available in F. Castelli's website (Castelli \& Kurucz 2003). For all the models we adopted an input metallicity of $[A / H]=-1.5 \mathrm{dex}$, according to the value derived by Hankey \& Cole (2011).

Initial atmospheric parameters were obtained from the Stetson (1994) optical photometry. First, $T_{\text {eff }}$ was derived from the $B-V$ colour using Alonso et al. (1999) and the reddening $E(B-V)=0.23$. Surface gravities $(\log (g))$ were obtained assuming a stellar mass of $0.8 M_{\odot}$ and a distance modulus of $(m-M)_{\mathrm{V}}=17.08$ (Harris 1996; 2010 edition). The bolometric correction $(\mathrm{BC})$ was derived by adopting the relation $\mathrm{BC}-T_{\mathrm{eff}}$

3 http://www.cosmic-1ab.eu/4dao/4dao.php 
Table 1. Coordinates, $V$ magnitude, radial velocities, and atmospheric parameters of IC 4499 stars observed with UVES.

\begin{tabular}{rccccccc}
\hline \hline ID & $\begin{array}{c}\text { RA(J2000) } \\
(\mathrm{deg})\end{array}$ & $\begin{array}{c}\text { Dec(J2000) } \\
(\mathrm{deg})\end{array}$ & $\begin{array}{c}V \\
(\mathrm{mag})\end{array}$ & $\begin{array}{c}v_{\mathrm{r}} \\
\left(\mathrm{km} \mathrm{s}^{-1}\right)\end{array}$ & $\begin{array}{c}T_{\text {eff }} \\
(\mathrm{K})\end{array}$ & $\begin{array}{c}\log g \\
(\mathrm{dex})\end{array}$ & $\begin{array}{c}\xi \mathrm{t} \\
\left(\mathrm{km} \mathrm{s}^{-1}\right)\end{array}$ \\
\hline $\mathrm{S} 354$ & 224.4198320 & -82.1760627 & 15.189 & $35.4 \pm 0.4$ & 4280 & 0.85 & 1.8 \\
S79 & 225.0212891 & -82.1874560 & 15.230 & $38.6 \pm 0.4$ & 4300 & 0.90 & 1.8 \\
$\mathrm{~S} 634$ & 224.9185887 & -82.2678076 & 15.267 & $37.1 \pm 0.4$ & 4350 & 0.70 & 2.1 \\
S81 & 225.0442379 & -82.1880345 & 15.588 & $37.0 \pm 0.4$ & 4430 & 0.90 & 1.7 \\
S639 & 224.9446419 & -82.2710076 & 15.683 & $36.2 \pm 0.4$ & 4400 & 0.75 & 1.6 \\
$\mathrm{~S} 664$ & 225.4816207 & -82.1793317 & 15.822 & $37.7 \pm 0.5$ & 4600 & 1.10 & 1.9 \\
\hline
\end{tabular}

Table 2. Abundance ratios for the observed stars.

\begin{tabular}{|c|c|c|c|c|c|c|c|c|c|c|c|c|c|c|c|c|}
\hline ID & {$[\mathrm{FeI} / \mathrm{H}]$} & $\sigma_{\text {rand }}$ & $\sigma_{\text {tot }}$ & {$[\mathrm{FeII} / \mathrm{H}]$} & $\sigma_{\text {rand }}$ & $\sigma_{\text {tot }}$ & {$[\mathrm{O} / \mathrm{Fe}]$} & $\sigma_{\text {rand }}$ & $\sigma_{\text {tot }}$ & {$[\mathrm{Na} / \mathrm{Fe}]$} & $\sigma_{\text {rand }}$ & $\sigma_{\text {tot }}$ & $\triangle \mathrm{NLTE}$ & {$[\mathrm{Mg} / \mathrm{Fe}]$} & $\sigma_{\text {rand }}$ & $\sigma_{\text {tot }}$ \\
\hline S354 & -1.64 & 0.01 & 0.07 & -1.57 & 0.04 & 0.09 & 0.45 & 0.04 & 0.09 & -0.09 & 0.03 & 0.09 & -0.07 & 0.40 & 0.04 & 0.09 \\
\hline S79 & -1.66 & 0.01 & 0.07 & -1.59 & 0.04 & 0.09 & 0.40 & 0.03 & 0.09 & -0.10 & 0.04 & 0.09 & -0.07 & 0.37 & 0.04 & 0.09 \\
\hline S634 & -1.70 & 0.01 & 0.08 & -1.62 & 0.02 & 0.08 & 0.34 & 0.05 & 0.08 & 0.23 & 0.03 & 0.08 & -0.10 & 0.27 & 0.04 & 0.08 \\
\hline S81 & -1.69 & 0.01 & 0.06 & -1.61 & 0.05 & 0.10 & 0.54 & 0.05 & 0.10 & -0.22 & 0.05 & 0.10 & -0.05 & 0.22 & 0.05 & 0.09 \\
\hline S639 & -1.56 & 0.01 & 0.07 & -1.52 & 0.05 & 0.07 & 0.33 & 0.03 & 0.07 & -0.21 & 0.04 & 0.07 & -0.07 & 0.30 & 0.03 & 0.07 \\
\hline S664 & -1.61 & 0.01 & 0.09 & -1.52 & 0.06 & 0.10 & 0.43 & 0.05 & 0.10 & -0.24 & 0.05 & 0.10 & -0.06 & 0.25 & 0.08 & 0.09 \\
\hline
\end{tabular}

Table 3. Estimated errors on abundances due to errors on atmospheric parameters for star S354.

\begin{tabular}{rcccccccc}
\hline \hline Element & $+\Delta T_{\text {eff }}$ & $+\Delta T_{\text {eff }}$ & $+\Delta \log (g)$ & $+\Delta \log (g)$ & $+\Delta \xi \mathrm{t}$ & $+\Delta \xi \mathrm{t}$ & $+\Delta[M / H]$ & $-\Delta[M / H]$ \\
\hline Fe I & 0.09 & -0.08 & 0.01 & 0.00 & -0.10 & 0.16 & -0.00 & 0.00 \\
Fe II & -0.06 & 0.06 & 0.09 & -0.09 & -0.05 & 0.08 & 0.03 & -0.03 \\
O I & -0.01 & 0.01 & 0.09 & -0.09 & -0.02 & 0.02 & 0.04 & -0.04 \\
Na I & 0.06 & -0.07 & -0.02 & 0.02 & -0.04 & 0.05 & -0.01 & 0.01 \\
$\mathrm{Mg}$ I & 0.06 & -0.06 & -0.02 & 0.02 & -0.10 & 0.14 & -0.01 & 0.01 \\
\hline
\end{tabular}

from Alonso et al. (1999). Finally, the initial micro-turbulence velocity $(\xi \mathrm{t})$ was set to $2 \mathrm{~km} \mathrm{~s}^{-1}$ for all stars. These atmospheric parameters were considered as initial estimates and were refined during the abundance analysis. $T_{\text {eff }}$ is adjusted until there is no trend between the abundance from Fe I lines and the excitation potential. The surface gravity is optimised in order to minimise the difference between the abundance derived from neutral and single ionised iron. Finally, $\xi \mathrm{t}$ is determined by erasing any trend between the abundance from Fe I and the reduced EW.

The mean abundances of Table 2 are computed by averaging the abundances of the surviving lines weighted by the uncertainty on the abundance as obtained from the EW error. The random errors ( $\sigma_{\text {rand }}$ in Table 2$)$ are computed as the dispersion of the mean normalised to the root mean square of the number of used lines. For those elements for which only one line is available, the error associated with the individual abundance estimate is taken as the internal error.

The errors due to the uncertainty in the atmospheric parameters are obtained varying atmospheric parameters one at the time, by $\Delta T_{\text {eff }}= \pm 70 \mathrm{~K}, \Delta \log (g)= \pm 0.2 \mathrm{dex}, \Delta \xi t= \pm 0.5 \mathrm{~km} \mathrm{~s}^{-1}$, and $\Delta[M / H]= \pm 0.1$ dex and redetermining abundances. Results are shown in Table 3 for the star S354, assumed to represent the entire sample. The total error $\left(\sigma_{\text {tot }}\right)$, listed in Table 2 is obtained by adding the errors in quadrature. Fe abundances were measured for all stars in our sample, whereas $\mathrm{O}$ and $\mathrm{Na}$ abundances are available only for four stars. Five stars have measured $[\mathrm{Mg} / \mathrm{Fe}]$ abundance ratios.

Corrections for departures from the LTE approximation are applied only for the $\mathrm{Na}$ lines, by interpolating on the grid of corrections calculated by Lind et al. (2011). In general these corrections are of the order of $\Delta \mathrm{NaI}_{\mathrm{LTE}-\mathrm{NLTE}}=-0.07 \mathrm{dex}$. Reference solar abundances are by Grevesse \& Sauval (1998) except for oxygen, for which we adopted the value provided by Caffau et al. (2008).

\section{Results}

\subsection{Photometric evidence of MPs}

To test the presence of MPs in IC 4499 we first used the pseudo-colour diagram $\left(m_{\mathrm{F} 336 \mathrm{~W}}, C_{275,336,438}\right)$ shown in Fig. 5 $\left(C_{275,336,438}=\left(m_{\mathrm{F} 275 \mathrm{~W}}-m_{\mathrm{F} 336 \mathrm{~W}}\right)-\left(m_{\mathrm{F} 336 \mathrm{~W}}-m_{\mathrm{F} 438 \mathrm{~W}}\right)\right)$, as it traces simultaneously the $\mathrm{OH}, \mathrm{NH}$ and $\mathrm{CH}$ molecular bands strength (e.g. Piotto et al. 2015; Milone et al. 2017). In this diagram we defined two fiducial lines (left panel of Fig. 5) following the bluest and reddest end of the RGB and we then verticalised the distribution of RGB stars in the magnitude range $18.2<m_{\mathrm{F} 336 \mathrm{~W}}<20.5$. The verticalised colour distribution $\left(\Delta_{C_{275,336,438}}\right)$ is clearly bimodal with two peaks at $\Delta_{C_{275.336 .438}} \sim 0.03$ and $\sim 0.14$ respectively (middle panel of Fig. 5). The detected bimodality is statistically significant, and a unimodal distribution can be rejected with a confidence level $>3 \sigma$ according to a Gaussian Mixture Models analysis (Muratov \& Gnedin 2010). This result represents the first evidence of the presence of MPs in IC 4499.

We classified the two sub-populations as FP and SP moving from red to blue colours as stars are expected to be progressively enhanced in $\mathrm{N}$ at decreasing $C_{275,336,438}$ colours. From the areas under the Gaussian functions, we computed the number ratios 

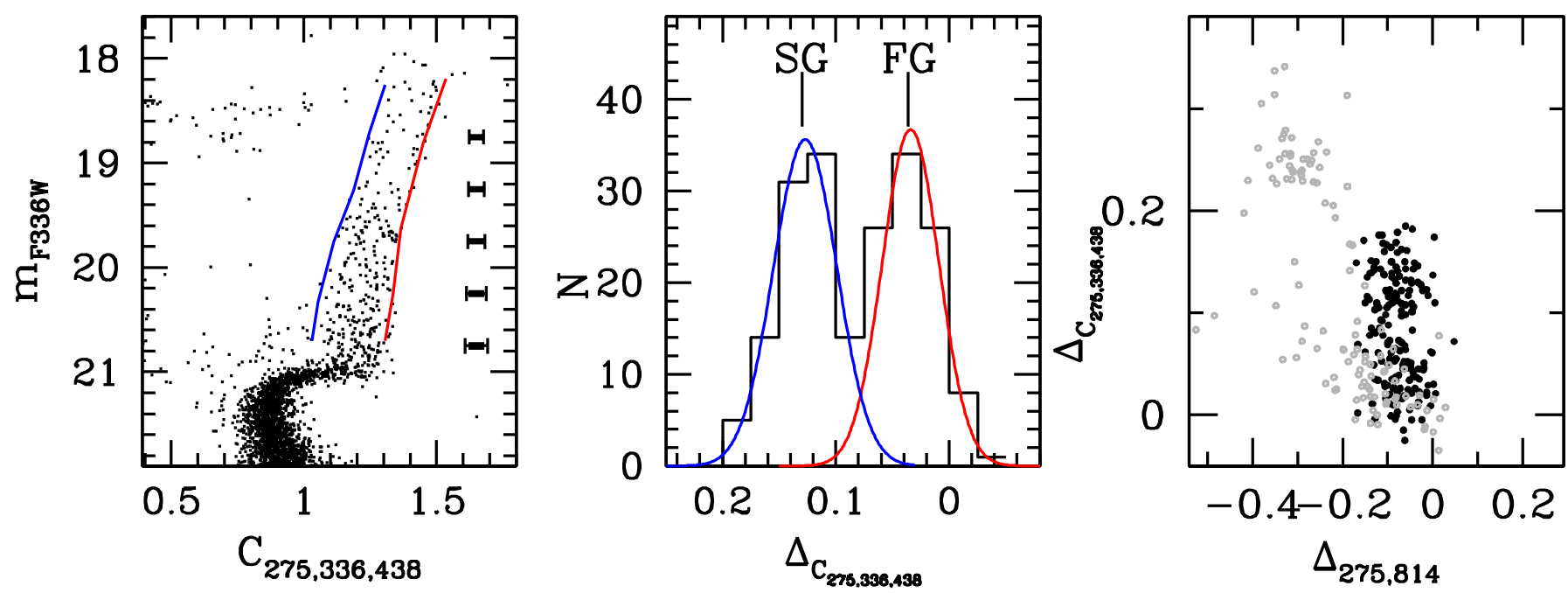

Fig. 5. Left panel: $\left(m_{\mathrm{F} 336 \mathrm{~W}}, C_{275,336,438}\right)$ pseudo-colour diagram of IC 4499. Typical photometric errors for different magnitude bins are also shown. Middle panel: verticalised pseudo-colour distribution $\Delta C_{275,336,438}$ of RGB stars in the magnitude range $18.2<m_{\mathrm{F} 336 \mathrm{~W}}<20.5$. Two main components can be clearly identified (SP and FP, in blue and red; respectively). Right panel: $\left(\Delta_{275,814}, \Delta C_{275,336,438}\right)$ colour-colour diagram ("chromosome map") of IC 4499. Overplotted for reference is shown also the chromosome map of NGC 288 (light grey).

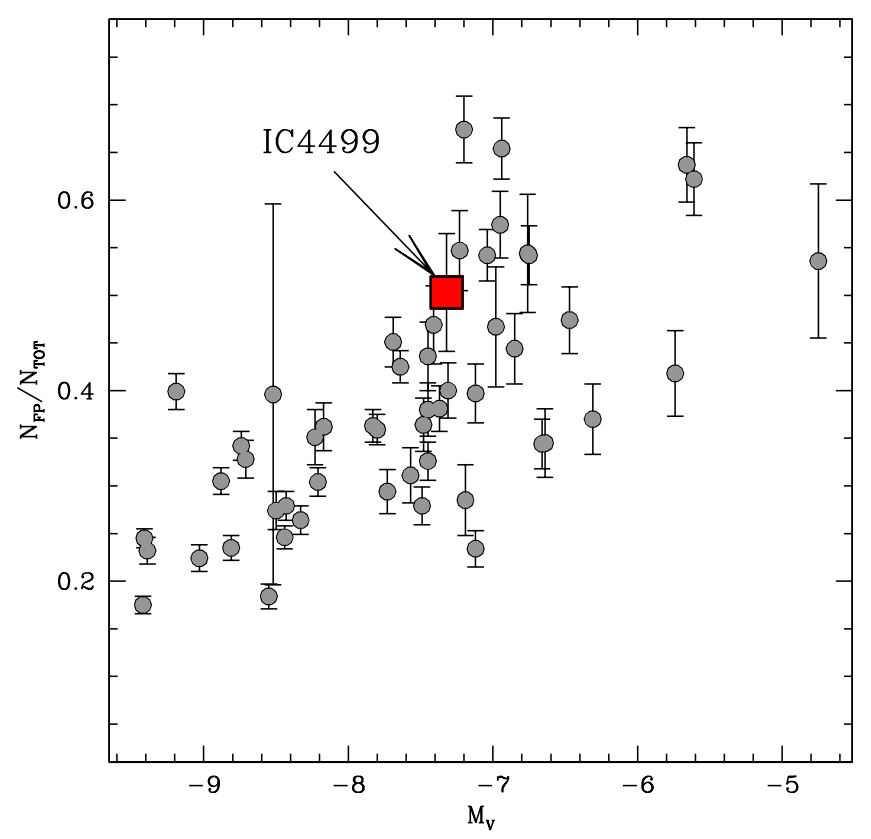

Fig. 6. Fraction of FP stars respect to the total number of RGB stars (counted in the same magnitude range) as a function of the absolute cluster magnitudes. IC 4499 is highlighted with a red square and it follows the general behaviour described by the other GCs observed within the HST UV Legacy Survey (Piotto et al. 2015; Milone et al. 2017).

between the sub-populations. We find that 97 stars can be attributed to the FP and 96 to the SP sub-sample, thus yielding $N_{\mathrm{FP}} / N_{\mathrm{TOT}}=0.503 \pm 0.062$, where $N_{\mathrm{TOT}}$ is the total number of RGB in the considered magnitude range. As shown in Fig. 6, given the absolute magnitude of IC $4499\left(M_{\mathrm{V}}=-7.32\right.$; Harris 1996), the derived value of $N_{\mathrm{FP}} / N_{\mathrm{TOT}}$ appears to be compatible with the general $\left(N_{\mathrm{FP}} / N_{\mathrm{TOT}}, M_{\mathrm{V}}\right)$ trend observed for other GCs analysed adopting the same observational strategy (instrument and filters) as for IC 4499.

We have also computed the $\left(\Delta_{275,814}, \Delta_{275,336,438}\right)$ diagram (the so-called "chromosome map"; Milone et al. 2017), which is shown in the right panel of Fig. 5. $\Delta_{275,814}$ was obtained by verticalising the distribution of RGB stars in the $\left(m_{\mathrm{F} 814 \mathrm{~W}}, m_{\mathrm{F} 275 \mathrm{~W}}-m_{\mathrm{F} 814 \mathrm{~W}}\right) \mathrm{CMD}$ in the same magnitude range used before. MPs are clearly separated also in this diagram. We also note that both FP and SP stars are well clumped in the chromosome map attaining very similar $\left(m_{\mathrm{F} 275 \mathrm{~W}}-m_{\mathrm{F} 814 \mathrm{~W}}\right)$ colours thus suggesting a very small (if any) He variation within each and among the two sub-populations (e.g. Milone et al. 2015; Lardo et al. 2018). This result is further confirmed by the relative colour distribution of FP and SP stars in the $\left(m_{\mathrm{F} 606 \mathrm{~W}}, m_{\mathrm{F} 606 \mathrm{~W}}-m_{\mathrm{F} 814 \mathrm{~W}}\right) \mathrm{CMD}$. In fact, we find that their average colour difference is well within the photometric errors along the RGB. For comparison, in Fig. 5 we also show the $\left(\Delta_{275,814}, \Delta_{275,336,438}\right)$ distribution for NGC 288, which is a well studied low-mass $\left(M_{\mathrm{V}}=-6.75\right.$; Harris 1996) cluster with similar metallicity to IC 4499 .

The intrinsic RGB widths both in $\left(\Delta_{275,814}\right)$ and $\left(\Delta_{275,336,438}\right)$ colour combinations were derived for stars 2 mag brighter than the TO in the $m_{\mathrm{F} 814 \mathrm{~W}}$ band. We subtracted photometric errors and potential residuals of the differential reddening corrections to the observed widths and we obtain $W_{C(275,336,438)}=0.195 \pm 0.009$ and $W_{275,814}=0.132 \pm 0.008$. Interestingly, Fig. 7 shows that the RGB width $W_{C(275,336,438)}$ of IC 4499 is significantly smaller than what observed in other clusters with similar metallicity and mass, thus suggesting a significantly smaller enhancement in $\mathrm{N}$ abundance. Only one cluster, namely NGC 6584, has a similar RGB width $\left(W_{C(275,336,438)}=0.195 \pm 0.009\right.$; Milone et al. 2017) in the same metallicity and mass range.

We have also derived the radial distribution of FP and SP sub-populations. To this aim, we first calculated the cluster centre $\left(C_{\text {grav }}\right)$ following the method reported by Dalessandro et al. (2018, see also Cadelano et al. 2017), which is based on direct star counts. Briefly, $C_{\text {grav }}$ has been determined following an iterative procedure that starts from a first-guess centre and computes the average of the coordinates ( $\alpha$ and $\delta$ ) of a sub-sample of stars located within a circle of radius $r$ and in a defined magnitude interval. The procedure stops when convergence is reached, that is when the newly determined centre coincides with the previous ones within an adopted tolerance limit. We repeated the procedure 27 times, using different values of $r$ and selecting stars in different 


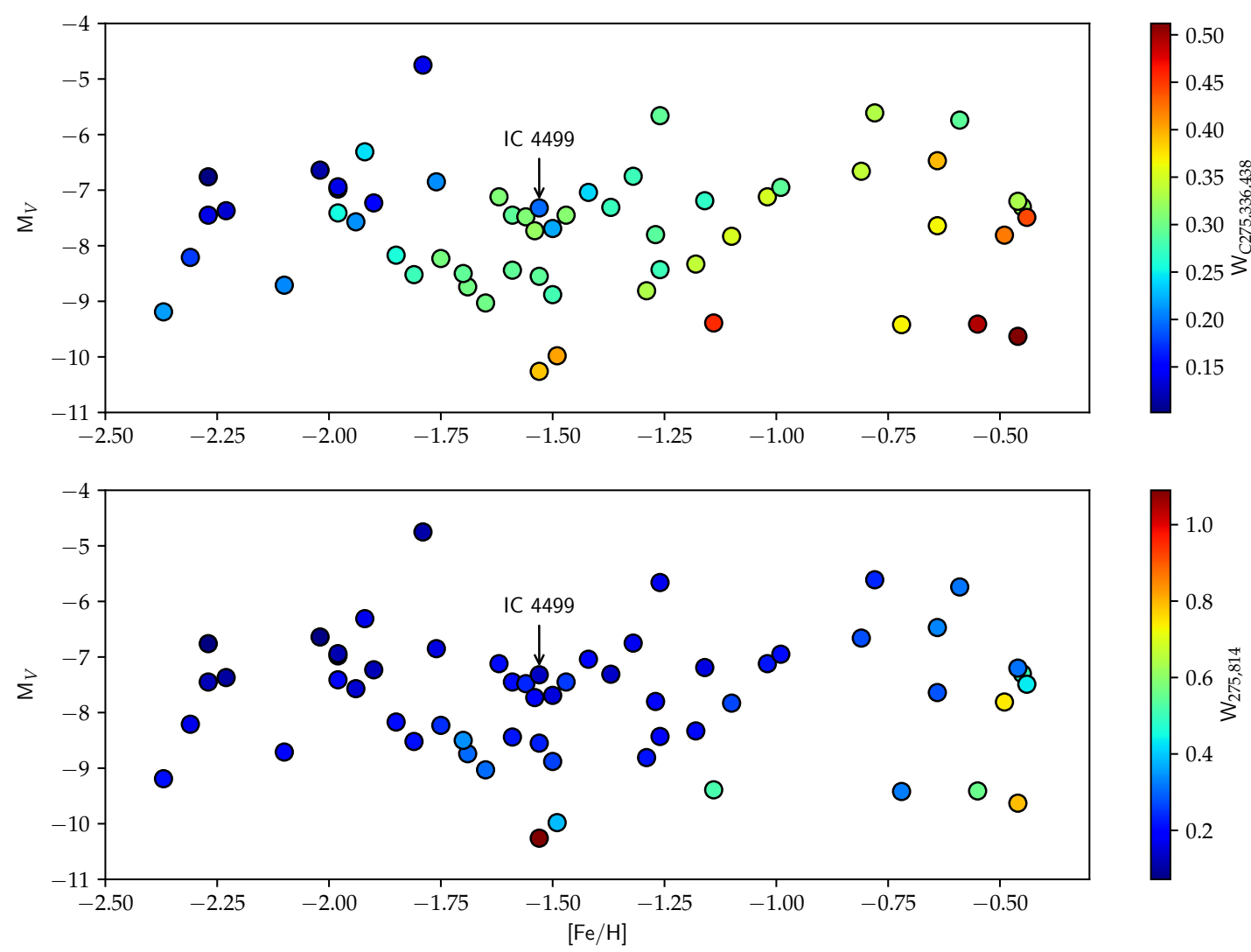

Fig. 7. Metallicity vs. integrated $V$ magnitude distribution of GCs observed within the HST UV Legacy Survey. Clusters are colour coded according to the values of $W_{C(275,336,438)}$ (top panel) and $W_{275,814}$ (bottom panel). IC 4499 is highlighted in both plots.

magnitude ranges, chosen as a compromise between having high enough statistics and avoiding spurious effects due to incompleteness and saturation. In particular, the radius $r$ has been chosen in the range of $85^{\prime \prime}-100^{\prime \prime}$ with a step of $5^{\prime \prime}$, thus guaranteeing that it is always larger than the literature core radius $r_{\mathrm{c}}=50.4$ (Harris 1996). For each radius $r$, we have explored six magnitude ranges, from $m_{\mathrm{F} 606 \mathrm{~W}} \geq 14$ (in order to exclude stars close to the saturation limit), down to $m_{\mathrm{F} 606 \mathrm{~W}}=22$, in steps of $0.3-0.2 \mathrm{mag}$. As the firstguess centre, we used the one quoted by Shawl \& White (1986). The final value adopted as $C_{\text {grav }}$ is the mean of the different values of $\alpha$ and $\delta$ obtained in the 27 explorations, and its uncertainty is their standard deviation. The derived $C_{\text {grav }}$ results to be located at $\alpha=15^{\mathrm{h}} 0^{\mathrm{m}} 18.749^{\mathrm{s}}$ and $\delta=-82^{\circ} 12^{\prime} 52.486^{\prime \prime}$, with a total uncertainty of about $2^{\prime \prime}$. This newly determined centre is $3.2^{\prime \prime}$ distant from the one quoted by Shawl \& White (1986).

The cumulative radial distributions of FP and SP stars with respect to $C_{\text {grav }}$ and the number ratio $N_{\mathrm{SP}} / N_{\mathrm{FP}}$ radial variations within the entire WFC3 FOV are shown in Fig. 8. SP stars are more centrally concentrated than FP. The Kolmogorov-Smirnov test gives a probability $P_{\mathrm{KS}} \sim 0.03$ that the two populations are extracted from the same parent population. However, it is important to stress that the HST data cover only $\sim 1 r_{\mathrm{h}}\left(r_{\mathrm{h}} \sim 102^{\prime \prime}\right.$; Harris 1996). As discussed in Dalessandro et al. (2018), radial distributions obtained over a limited area $\left(<1.5-2 \times r_{\mathrm{h}}\right)$ might not be representative of the overall relative distribution and therefore should be taken with some caution.

\subsection{Abundance results}

The chemical analysis of IC 4499 provides a mean metallicity of $[\mathrm{FeI} / \mathrm{H}]=-1.64(\sigma=0.05) \mathrm{dex}$ and $[\mathrm{FeII} / \mathrm{H}]=-1.57$ $(\sigma=0.04)$ dex. This value is slightly lower (but still consistent)

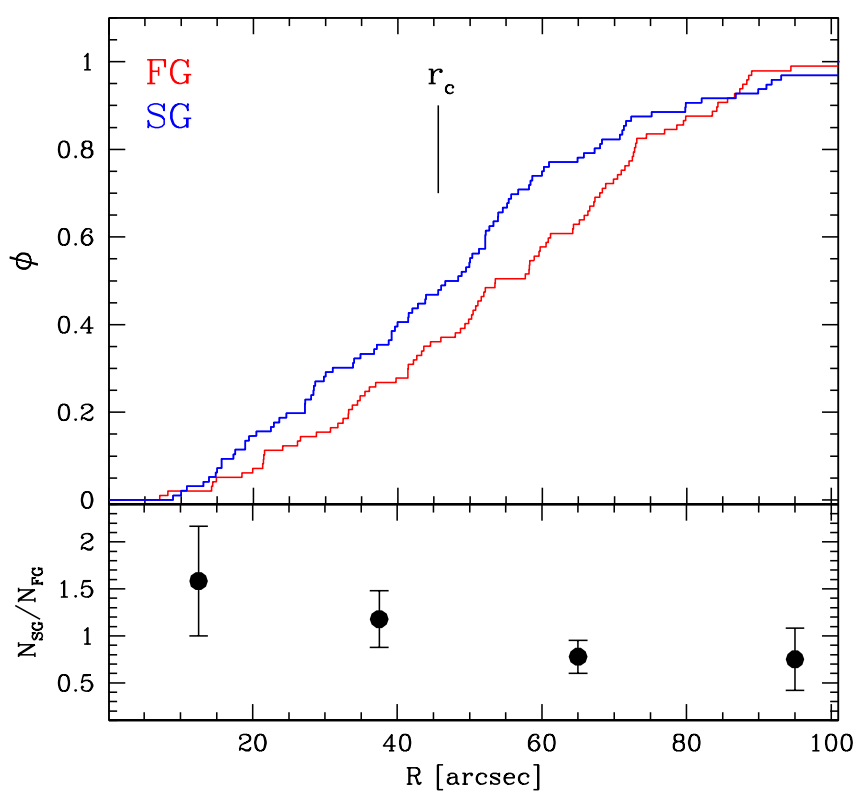

Fig. 8. Upper panel: cumulative radial distribution of FP (red), and SP (blue) stars. Lower panel: ratio between SP and FP stars as a function of the distance from the cluster centre. The position of the core radius $\left(r_{\mathrm{c}}\right)$ is also shown.

than previous derivations by Hankey \& Cole (2011) who measured $[\mathrm{Fe} / \mathrm{H}]=-1.52 \pm 0.12 \mathrm{dex}$. However, these analyses were based on low-resolution spectra around the calcium triplet feature that is more prone to uncertainties and to systematics related to the adopted calibrations. 


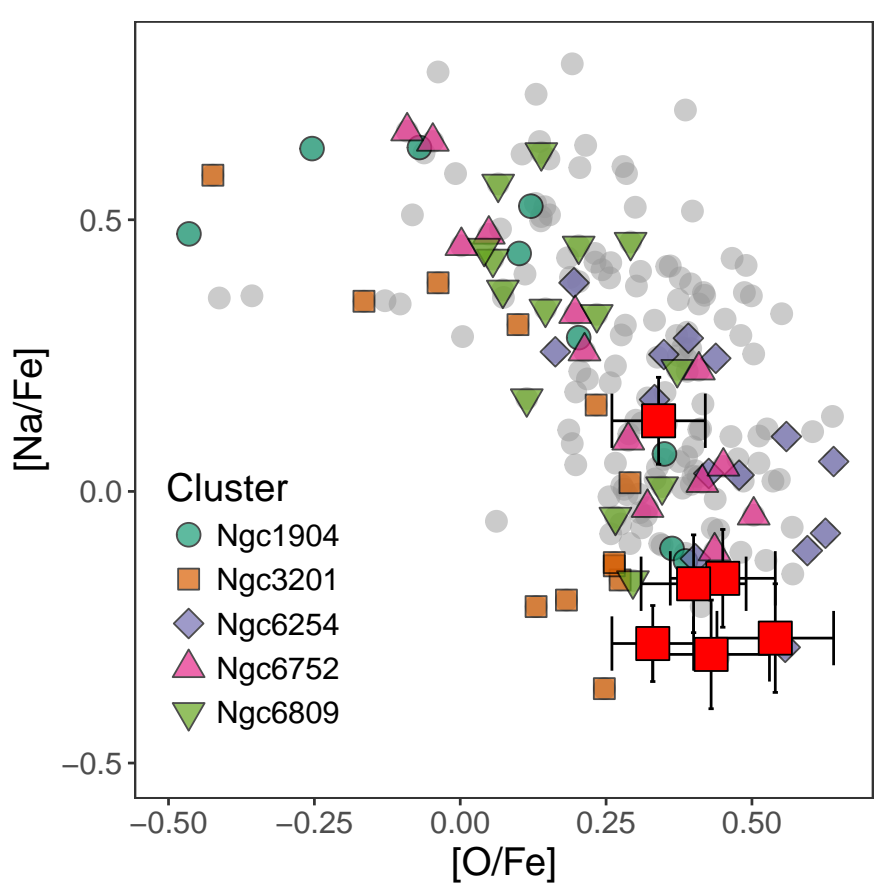

Fig. 9. IC4499 $[\mathrm{Na} / \mathrm{Fe}]$ and $[\mathrm{O} / \mathrm{Fe}]$ abundance ratios (red squares) are over-plotted to the homogeneous data from the Carretta et al. (2009) survey of ancient Galactic globulars (grey circles). Clusters with similar metallicity as IC 4499 are also highlighted (see legend).

The mean $[\mathrm{O} / \mathrm{Fe}]=0.42(\sigma=0.07)$ dex, with no indication of intrinsic spreads. Five out of six stars have also very similar $\mathrm{Na}$ abundances within the uncertainties. On the other hand, one star (S634 - see Table 2) has higher Na abundance by $\sim 0.4$ dex, whereas both $\mathrm{O}$ and $\mathrm{Mg}$ abundances are consistent with the other stars in the sample. This behaviour is consistent with the $\mathrm{Na}-\mathrm{O}$ anti-correlation observed in other GCs as shown in Fig. 9 and obtained by Carretta et al. (2009). While in GCs with global extended $\mathrm{Na}-\mathrm{O}$ anti-correlation, both $[\mathrm{O} / \mathrm{Fe}]$ and $[\mathrm{Na} / \mathrm{Fe}]$ display large ranges, for small $[\mathrm{Na} / \mathrm{Fe}]$ variations around the field star area, $[\mathrm{O} / \mathrm{Fe}]$ stay almost constant, as for the case of IC 4499 (red squares in Fig. 9). This result further confirms the evidence that IC 4499 does host MPs. The detection of only one Na enriched star (corresponding to $16 \pm 18 \%$ of the sample) does not match the expectations based on photometric results of almost equipopulated sub-populations. However, we note that spectroscopic targets are distributed well outside the HST FOV, where the $\mathrm{SP} / \mathrm{FP}$ ratio appears to be significantly lower than in the central regions.

Consistently with what constrained by using UV photometry, IC 4499 shows the least extended $\mathrm{Na}-\mathrm{O}$ anticorrelation compared to clusters with similar metallicity. However, the significance of such a comparison is limited by the low-number statistics of our sample.

For $\mathrm{Mg}$, we find a mean abundance of $[\mathrm{Mg} / \mathrm{Fe}]=0.30$ $(\sigma=0.07)$ that well compares with the mean $[\mathrm{Mg} / \mathrm{Fe}]$ abundance ratio measured by Carretta et al. (2009) in NGC 6254, NGC 6752, and NGC 6809. As for O, Mg does not show intrinsic abundance variations.

\section{Summary and conclusions}

IC 4499 was suggested to be one of the very few old Galactic globular clusters to not show MPs (Walker et al. 2011). This result was based on multi-band ground-based photometry, targeting a limited number of stars and likely dominated by the FP sub-populations as observations were mainly biased towards the external regions of the cluster. In this study, we present the first evidence that IC 4499 does host MPs with light-element abundance variations.

Our analysis is based on both high-resolution photometric and spectroscopic data. The UV and optical HST photometry shows that the RGB of IC 4499 clearly splits in two sequences, which are also well identified in the so called chromosome map $\left(\left(\Delta_{275,814}\right),\left(\Delta_{C 275,336,438}\right)\right.$ diagram $)$ thus suggesting they have different light-element abundances. This result is qualitatively confirmed by the $\mathrm{Na}$ abundance distribution. In fact, we find that one star has $\mathrm{Na}$ abundance compatible with being $\mathrm{SP}$ as it is enriched by $\sim 0.4$ dex. Conversely both $\mathrm{O}$ and $\mathrm{Mg}$ do not show any evidence for internal variations.

Interestingly, we find that the fraction of SP with respect to FP stars well fits the trend with the present-day cluster mass observed for other Galactic GCs (e.g. Milone et al. 2017). On the contrary, the width of the RGB in both $\left(\Delta_{275,814}\right)$ and $\left(\Delta_{C 275,336,438}\right)$ - which are proxies for the $Y$ and $N$ enhancement, respectively (e.g. Milone et al. 2015; Lardo et al. 2018) - are significantly smaller than the values derived for old Galactic globulars with similar metallicity and mass by Milone et al. (2017). A similar indication is also suggested by the $\mathrm{Na}$ abundance distribution, although it is not conclusive because of the small sample size.

Hankey \& Cole (2011) suggested a possible extra-Galactic origin of IC 4499. In fact, based on the mean radial velocity of the system, the authors found a possible connection with the Monoceros ring, which would eventually suggest that IC 4499 formed in the disrupted Canis Major dwarf galaxy (Helmi et al. 2003; Martin et al. 2005). In this context, it would be tempting to speculatively connect the moderate $\mathrm{N}$ and $\mathrm{Na}$ abundance variations observed in IC 4499 with its extra-Galactic origin. GCs formed in the Sagittarius dwarf galaxy and Fornax typically show small variations in terms of $\mathrm{Na}$ and $\mathrm{O}$ (with the obvious exception of the very massive system M 54; Carretta et al. 2014). In addition, they tend to show less extended horizontal branch morphologies (so possibly smaller He spreads) and redder farUV indexes than genuine Milky-Way GCs with similar mass and metallicity (Dalessandro et al. 2012).

While this trend is surely fascinating and possibly suggestive of a link between the environment and the efficiency of light-element enrichment a cluster can undergo, at the present stage it is hard to firmly disentangle it from the contribution of other key parameters, such as cluster mass and age. Moreover, a robust distinction between genuine Milky-Way and accreted systems is of crucial importance in this context. The exquisite astrometric performance of Gaia will definitely help to firmly constrain the extra-Galactic origin of some GCs and to unveil possible new accreted systems (see for example Myeong et al. 2018), thus possibly shedding new light on the actual role played by the environment on the properties of MPs.

Acknowledgements. The authors thank the referee for the careful reading of the paper. ED acknowledges financial support from the Leverhulme Trust Visiting Professorship Programme VP2-2017-030. ED is also grateful for the warm hospitality of LJMU where part of this work was performed. CL acknowledges financial support from the Swiss National Science Foundation (Ambizione grant PZ00P2_168065). NB gratefully acknowledges funding from the ERC under the European Union's Horizon 2020 research and innovation programme via the ERC Consolidator Grant Multi-Pop (grant agreement number 646928, PI Bastian). NB is a Royal Society University Research Fellow. 


\section{References}

Alonso, A., Arribas, S., \& Martínez-Roger, C. 1999, A\&AS, 140, 261 Bastian, N., Lamers, H. J. G. L. M., de Mink, S. E., et al. 2013, MNRAS, 436, 2398

Bellini, A., \& Bedin, L. R. 2009, PASP, 121, 1419

Bellini, A., Anderson, J., van der Marel, R. P., et al. 2014, ApJ, 797, 115

Bragaglia, A., Carretta, E., D’Orazi, V., et al. 2017, A\&A, 607, A44

Cadelano, M., Dalessandro, E., Ferraro, F. R., et al. 2017, ApJ, 836, 170

Caffau, E., Ludwig, H.-G., Steffen, M., et al. 2008, A\&A, 488, 1031

Carretta, E., Bragaglia, A., Gratton, R., \& Lucatello, S. 2009, A\&A, 505, 139

Carretta, E., Bragaglia, A., Gratton, R. G., et al. 2010, A\&A, 516, A55

Carretta, E., Bragaglia, A., Gratton, R. G., et al. 2014, A\&A, 561, A87

Casagrande, L., \& VandenBerg, D. A. 2014, MNRAS, 444, 392

Castelli, F., \& Kurucz, R. L. 2003, in Modelling of Stellar Atmospheres, eds., N., Piskunov, W. W., Weiss, \& D. F., Gray, IAU Symp., 210, 20

Chung, C., Yoon, S.-J., \& Lee, Y.-W. 2011, ApJ, 740, L45

D’Antona, F., Vesperini, E., D’Ercole, A., et al. 2016, MNRAS, 458, 2122

Dotter, A., Sarajedini, A., \& Anderson, J. 2011, ApJ, 738, 74

Dalessandro, E., Schiavon, R. P., Rood, R. T., et al. 2012, AJ, 144, 126

Dalessandro, E., Ferraro, F. R., Massari, D., et al. 2013, ApJ, 778, 135

Dalessandro, E., Lapenna, E., Mucciarelli, A., et al. 2016, ApJ, 829, 77

Dalessandro, E., Cadelano, M., Vesperini, E., et al. 2018, ApJ, 859, 15

Decressin, T., Meynet, G., Charbonnel, C., Prantzos, N., \& Ekström, S. 2007, A\&A, 464, 1029

Denissenkov, P. A., \& Hartwick, F. D. A. 2014, MNRAS, 437, L21

D’Ercole, A., Vesperini, E., D’Antona, F., McMillan, S. L. W., \& Recchi, S. 2008, MNRAS, 391, 825

Gieles, M., Charbonnel, C., Krause, M. G. H., et al. 2018, MNRAS,

Grevesse N., \& Sauval A. J. 1998, Space Sci. Rev., 85, 161

Hankey W. J., \& Cole A. A. 2011, MNRAS, 411, 1536

Harris, W. E. 1996, AJ, 112, 1487

Helmi, A., Navarro, J. F., Meza, A., Steinmetz, M., \& Eke, V. R. 2003, ApJ, 592 , L25
Kupka, F., Piskunov, N., Ryabchikova, T. A., Stempels, H. C., \& Weiss, W. W. 1999, A\&AS, 138, 119

Lardo, C., Salaris, M., Bastian, N., et al. 2018, A\&A, 616, A168

Larsen, S. S., Strader, J., \& Brodie, J. P. 2012, A\&A, 544, L14

Larsen, S. S., Brodie, J. P., Wasserman, A., \& Strader, J. 2018, A\&A, 613, A56

Lind, K., Asplund, M., Barklem, P. S., \& Belyaev, A. K. 2011, A\&A, 528, A103

Martin, N. F., Ibata, R. A., Conn, B. C., et al. 2005, MNRAS, 362, 906

Martocchia, S., Cabrera-Ziri, I., Lardo, C., et al. 2018, MNRAS, 473, 2688

Massari, D., Bellini, A., Ferraro, F. R., et al. 2013, ApJ, 779, 81

Milone, A. P., Marino, A. F., Piotto, G., et al. 2015, ApJ, 808, 51

Milone, A. P., Piotto, G., Renzini, A., et al. 2017, MNRAS, 464, 3636

Monaco, L., Villanova, S., Carraro, G., Mucciarelli, A., \& Moni Bidin, C. 2018, A\&A, 616, A181

Mucciarelli, A. 2013, ArXiv e-prints [arXiv: 1311.1403]

Mucciarelli, A., Carretta, E., Origlia, L., \& Ferraro, F. R. 2008, AJ, 136, 375

Mucciarelli, A., Origlia, L., Ferraro, F. R., \& Pancino, E. 2009, ApJ, 695, L134

Mucciarelli, A., Pancino, E., Lovisi, L., Ferraro, F. R., \& Lapenna, E. 2013, ApJ, 766, 78

Muratov, A. L., \& Gnedin, O. Y. 2010, ApJ, 718, 1266

Myeong, G. C., Evans, N. W., Belokurov, V., Sanders, J. L., \& Koposov, S. E. 2018, ApJ, 863, L28

Niederhofer, F., Bastian, N., Kozhurina-Platais, V., et al. 2017, MNRAS, 465, 4159

Piotto, G., Milone, A. P., Bedin, L. R., et al. 2015, AJ, 149, 91

Schiavon, R. P., Caldwell, N., Conroy, C., et al. 2013, ApJ, 776, L7

Shawl, S. J., \& White, R. E. 1986, AJ, 91, 312

Stetson, P. B. 1987, PASP, 99, 191

Stetson, P. B. 1994, PASP, 106, 250

Stetson, P. B., \& Pancino, E. 2008, PASP, 120, 1332

Villanova, S., Geisler, D., Carraro, G., Moni Bidin, C., \& Muñoz, C. 2013, ApJ, 778,186

Walker, A. R., Kunder, A. M., Andreuzzi, G., et al. 2011, MNRAS, 415, 643 